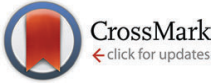

Cite this: Phys. Chem. Chem. Phys., $2016,18,18353$

Received 5th May 2016, Accepted 13th June 2016

DOI: $10.1039 / c 6 c p 03029 g$

www.rsc.org/pccp

\section{Secondary brown carbon formation via the dicarbonyl imine pathway: nitrogen heterocycle formation and synergistic effects $\dagger$}

\author{
C. J. Kampf, ${ }^{\star a b}$ A. Filippi, ${ }^{a b}$ C. Zuth, ${ }^{a}$ T. Hoffmann ${ }^{a}$ and T. Opatz
}

Dicarbonyls are known to be important precursors of so-called atmospheric brown carbon, significantly affecting aerosol optical properties and radiative forcing. In this systematic study we report the formation of light-absorbing nitrogen containing compounds from simple 1,2-, 1,3-, 1,4-, and 1,5dicarbonyl + amine reactions. A combination of spectrophotometric and mass spectrometric techniques was used to characterize reaction products in solutions mimicking atmospheric particulates. Experiments with individual dicarbonyls and dicarbonyl mixtures in ammonium sulfate and glycine solutions demonstrate that nitrogen heterocycles are common structural motifs of brown carbon chromophores formed in such reaction systems. 1,4- and 1,5-dicarbonyl reaction systems, which were used as surrogates for terpene ozonolysis products, showed rapid formation of light-absorbing material and products with absorbance maxima at $\sim 450 \mathrm{~nm}$. Synergistic effects on absorbance properties were observed in mixed (di-)carbonyl experiments, as indicated by the formation of a strong absorber in ammonium sulfate solutions containing acetaldehyde and acetylacetone. This cross-reaction oligomer shows an absorbance maximum at $385 \mathrm{~nm}$, relevant for the actinic flux region of the atmosphere. This study demonstrates the complexity of secondary brown carbon formation via the imine pathway and highlights that cross-reactions with synergistic effects have to be considered an important pathway for atmospheric BrC formation.

\section{Introduction}

One of the main open questions regarding organic compounds in atmospheric chemistry today is related to the formation of light-absorbing compounds and the occurrence of so called brown carbon (BrC). ${ }^{1,2}$ While organic compounds in ambient fine particles have been assumed for decades not to absorb solar radiation, thus resulting in a net cooling effect on climate, ${ }^{3,4}$ it is now generally accepted that a continuum of light-absorbing carbonaceous species is present in fine aerosols. ${ }^{5,6}$

Brown carbon (BrC) is a fraction of organic material in atmospheric particulates that absorbs light with a strong wavelengths dependence $\left(\lambda^{-2}-\lambda^{-6}\right),{ }^{1,2,7}$ thus showing an increasing absorption from the visible to the ultraviolet wavelengths. ${ }^{1,8,9}$

\footnotetext{
${ }^{a}$ Institut für Anorganische und Analytische Chemie, Johannes Gutenberg-Universität Mainz, 55128 Mainz, Germany. E-mail: kampfc@uni-mainz.de

${ }^{b}$ Abteilung für Multiphasenchemie, Max-Planck-Institut für Chemie, 55128 Mainz, Germany

${ }^{c}$ Institut für Organische Chemie, Johannes Gutenberg-Universität Mainz, 55128 Mainz, Germany

$\dagger$ Electronic supplementary information (ESI) available: UV/Vis spectra, HPLCDAD chromatograms, HRMS/MS spectra, reference compound analysis. See DOI: 10.1039/c6cp03029g
}

Radiative forcing of $\mathrm{BrC}$ has been estimated to account for $27-70 \%$ of the black carbon (BC) forcing in a recent model study. ${ }^{10}$ The chemistry of brown carbon formation in the atmosphere and its impact on aerosol optical properties have been discussed in detail in recent review articles. ${ }^{2,11}$ Briefly, BrC can originate from primary, i.e., primary organic aerosols (POA) emitted from biomass and biofuel combustion, and from secondary sources (see ref. 2 and 11 and references therein). Formation mechanisms of secondary BrC include, amongst others, the nitration of aromatic compounds, ${ }^{12-15}$ formation of light-absorbing higher molecular weight oligomers, e.g., by acid catalyzed aldol condensation reactions, ${ }^{16-18}$ and reactions of ammonia $\left(\mathrm{NH}_{3}\right)$ or amino acids with (di-)carbonyl species in secondary organic aerosols (SOA). ${ }^{16,17,19-36}$ Recent modeling studies suggest that $\mathrm{BrC}$ could contribute significantly or sometimes dominate overall aerosol absorption at specific wavelengths. ${ }^{9,37}$ Furthermore, some BrC constituents have been found to act as photosensitizers in SOA, therefore promoting further photoreactions including radical chemistry within the particle phase and influencing aqueous phase chemistry. ${ }^{38}$

The formation of light-absorbing products from (di-)carbonyl + ammonia or amino acid reactions have received increasing attention over the past years. Glyoxal (Gly) and methylglyoxal 
(MGly) are the two simplest 1,2- or $\alpha$-dicarbonyls and are formed in atmospheric oxidation processes of a number of biogenic and anthropogenic volatile organic compounds (VOCs). Thus, they occur ubiquitously in the atmosphere. ${ }^{39,40}$ Due to the hydration of its carbonyl functions and "salting-in" effects by sulfate ions in the aerosol particle phase, glyoxal has been shown to partition efficiently to the particle phase. ${ }^{41-45}$ Inorganic ammonium salts, or ammonium ions respectively, have been shown to catalyze carbonyl compound self-reactions like hemiacetal oligomer formation and aldol condensation..$^{20,46-49}$ Upon reactions with ammonia, which is present in aqueous aerosols in equilibrium with ammonium ions depending on $\mathrm{pH}$, Gly and MGly can form nitrogen heterocycles, i.e., imidazoles, and open chain nitrogen containing compounds, which have been identified as major light-absorbing species in these reactions..$^{23,25,27,35,50}$

Recently, browning of SOA from biogenic (isoprene, mono-, and sesquiterpenes) and anthropogenic (alkane, aromatic, $\mathrm{PAH}$ ) precursors collected on filters was observed upon exposure to ammonia gas. ${ }^{17,22,28,51}$ The change in absorption properties was attributed to the formation of light-absorbing species from the reactions of ammonia and specific carbonyl intermediates of the VOC oxidation reactions, e.g., ketolimononaldehyde, while other carbonyl intermediates, e.g., pinonaldehyde (a first generation oxidation product of $\alpha$-pinene) or limononaldehyde (a first generation oxidation product of limonene) did not form BrC. ${ }^{31}$

Organic matrices were found to affect the formation rates of light-absorbing compounds from $\alpha$-dicarbonyls in aqueous ammonium salt solutions, lowering the rates by up to one order of magnitude for high organic matrix to sulfate ratios. ${ }^{52}$ Further, BrC chromophores are subject to photo-bleaching upon irradiation, with atmospheric lifetimes of minutes to a few hours. ${ }^{53}$ However, BrC constituents such as the glyoxalderived $1 \mathrm{H}$-imidazole-2-carbaldehyde (IC) can act as photosensitizers, which are able to induce SOA growth due to a light-induced reactive uptake of volatile organic compounds. ${ }^{38}$

In this study, we focus on the investigation of chemical mechanisms and identification of light-absorbing products in secondary BrC formation. Mono-, 1,2-, 1,3-, 1,4-, and 1,5dicarbonyl compounds (dialdehydes, ketoaldehydes, and diketones) were used as BrC precursors. Ammonia (ammonium sulfate solutions) and the amino acid glycine served as amine components, which are likely to be encountered in atmospheric droplets. Experiments were performed in bulk solution experiments, including investigations of competitive reactions in mixed (di-)carbonyl systems, mimicking atmospheric particulates. The overall absorbance behavior of the reaction mixtures was monitored using UV/Vis spectrophotometry. Product structure identification was performed using High Performance Liquid Chromatography (HPLC) coupled with Diode Array Detection (DAD) and Mass Spectrometry (Electrospray Ionization Ion Trap Tandem Mass Spectrometry, ESI-MS ${ }^{2}$ ), as well as Ultra High Performance Liquid Chromatography (UHPLC) coupled with Orbitrap Mass Spectrometry (High Resolution Tandem Mass Spectrometry, ESI-HRMS ${ }^{2}$ ). The combination of HPLC-DADESI-MS $^{2}$ and UHPLC-ESI-HRMS ${ }^{2}$ provides a powerful tool for tentative structure elucidation of light-absorbing compounds in complex reaction systems.

\section{Materials and methods}

\section{Chemicals and materials}

Ammonium sulfate (AS, >99\%), glyoxal (Gly, 40 wt\% solution) and methylglyoxal (MGly, $40 \mathrm{wt} \%$ solution) were obtained from Acros organics; butanedione (BD, >97\%), acetylacetone (AcAc, $>99.5 \%$ ), hexanedione (HD, >98\%), glutaraldehyde (GA, $25 \mathrm{wt} \%$ solution), acetaldehyde (AcA, >99.9\%) and glycine ( $>99 \%$ ) from Sigma Aldrich; sodium hydroxide monohydrate from Merck KGaA; and hydrochloric acid ( $>37 \mathrm{wt} \%$ solution) from Appli Chem GmbH. All chemicals were used without further purification. Ultrapure water was obtained using an ELGA Purelab Option Q7/15 (Elga Veolia, Germany).

\section{Aerosol mimicry experiments}

In an approximation of an intermediate case between (over-)saturated SOA particle and low concentration cloud droplet conditions with regards to solute concentrations, $1 \mathrm{M}$ solutions of the respective dicarbonyls were prepared in $1 \mathrm{M}$ AS $(10 \mathrm{~mL}$ total volume) or $0.5 \mathrm{M}$ Gly. For each dicarbonyl/AS system, three solutions were prepared with an initial $\mathrm{pH}$ of 3-4. For dicarbonyl/ Gly systems two solutions were prepared with initial $\mathrm{pH}$ values of 3-5. Initial and final $\mathrm{pH}$ was monitored using a $\mathrm{pH}$ sensor (Multi 350 i with a Sentix electrode, WTW, Germany). Additionally, cross reactions of selected dicarbonyls were investigated in $1 \mathrm{M}$ AS solutions, which contained $1 \mathrm{M}$ concentrations of the following reactants (initial pH of 3-4): AcA + AcAc, Gly + MGly, Gly + AcAc, and Gly + MGly + GA. Reaction mixtures were stirred (Multistirrer 15, Fisher Scientific, Germany) in a closed screw cap vial wrapped in aluminum foil. The reaction systems studied in this work are summarized in Table 1.

For this study, sample aliquots taken after $0.5 \mathrm{~h}$ and $144 \mathrm{~h}$, which indicates the upper end of the atmospheric lifetime of an SOA particle, were analyzed. Further samples were collected at $1.5,2.5,4.5,5.5$ and $6.5 \mathrm{~h}$ reaction time and afterwards another sample was taken per day up to the total reaction time of 7 days

Table 1 Overview of reaction systems studied in this work with initial and final pH. A detailed list is given in Table S1 in the ESI

\begin{tabular}{|c|c|c|c|}
\hline Carbonyl component (1 M) & Amine component & Initial pH & Final pH \\
\hline $\mathrm{Gly}^{a}, \mathrm{MGly}^{b}, \mathrm{BD}^{c}, \mathrm{HD}^{d}, \mathrm{AcAc}^{e}, \mathrm{GA}^{f}$ & $\mathrm{AS}^{g}(1 \mathrm{M})$ & $3.2-4.2$ & $2.3-3.9$ \\
\hline Gly, MGly, BD, HD, AcAc, GA & glycine $^{h}(0.5 \mathrm{M})$ & $3.5-4.9$ & $2.9-3.9$ \\
\hline $\mathrm{AcA}^{\mathrm{i}}+\mathrm{AcAc}, \mathrm{Gly}+\mathrm{AcAc}, \mathrm{Gly}+\mathrm{MGly}, \mathrm{Gly}+\mathrm{MGly}+\mathrm{GA}$ & AS $(1 \mathrm{M})$ & $2.8-3.8$ & $1.7-2.6$ \\
\hline
\end{tabular}


for planned product kinetic studies totaling to 154 sample collections. At each sampling time, three $100 \mu \mathrm{L}$ aliquots were taken from the respective reaction mixture, corresponding to 462 collected samples. For dicarbonyl/AS reactions, the aliquots were mixed with $800 \mu \mathrm{L} \mathrm{MeOH}$ to precipitate unreacted AS and stop the reaction. After centrifugation (4 min/9000 rpm, Minispin, Eppendorf, Germany), $800 \mu \mathrm{L}$ of the supernatant were transferred to a $1.5 \mathrm{~mL}$ Eppendorf vial and stored at $-20{ }^{\circ} \mathrm{C}$ until analysis. Control samples analyzed directly after preparation and after one week of storage showed no significant difference in product peak areas ( $<5 \%$ deviation). For dicarbonyl/ Gly reactions, the aliquots were mixed with $800 \mu \mathrm{L}$ water and immediately frozen at $-20{ }^{\circ} \mathrm{C}$ until analysis.

\section{UV/Vis spectrophotometry}

UV/Vis absorption of the samples was analyzed using a Perkin Elmer - Lambda 25 UV-Vis spectrometer. The measurements were executed in disposable $70 \mu \mathrm{L} \mathrm{UV-cuvettes} \mathrm{(BRAND} \mathrm{GmbH} \mathrm{-}$ Wertheim). Due to (visible) differences in overall absorbance, two different dilutions for each sample were prepared and analyzed (dilution $1=1: 167$ and dilution $2=1: 50$ ).

All analyses were performed at room temperature and ambient pressure. A cuvette with $500 \mu \mathrm{L}$ water was used as reference sample. The instrument settings were: $1 \mathrm{~nm}$ slit width, $1 \mathrm{~nm}$

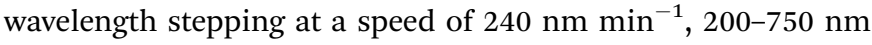
wavelength range.

\section{HPLC-DAD-ESI-MS ${ }^{2}$ measurements}

HPLC-DAD-ESI-MS was used to separate and characterize the products of the aforementioned reaction systems. Samples were analyzed using an HCT-Plus ion trap mass spectrometer (Bruker Daltonics GmbH, Bremen, Germany). The device was equipped with an HPLC-System (Agilent 1100 series, auto sampler, gradient pump, degasser and diode array detector (DAD), Agilent technologies GmbH, Germany) and an Atlantis T3 $150 \mathrm{~mm} \times 2.0$ column with $3 \mu \mathrm{m}$ particle size (Waters, Germany). Eluents were ultrapure water with $2 \%$ acetonitrile (A) and acetonitrile with $2 \%$ water (B). The injection volume was $10 \mu \mathrm{L}$. The gradient of the mobile phase, with a flow rate of

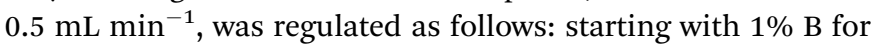
$2 \mathrm{~min}$, increasing to $75 \% \mathrm{~B}$ in $6 \mathrm{~min}$, isocratic for $4 \mathrm{~min}$, and decreasing to $1 \% \mathrm{~B}$ in $1 \mathrm{~min}$, followed by a re-equilibration time of $7 \mathrm{~min}$. The HPLC system was connected to the MS via the DAD equipped with a $500 \mathrm{~nL}$ flow cell $(1 \mathrm{~cm}$ optical path length). The DAD was operated with a slit width of $2 \mathrm{~nm}$ and was set to acquire a spectrum between 200 and $800 \mathrm{~nm}$ in steps of $2 \mathrm{~nm}$ every $2 \mathrm{~s}$. Mass spectrometric analyses were performed operating the ESI source in positive ion mode under the following conditions: 30 psi nebulizer pressure $\left(N_{2}\right), 10 \mathrm{~L} \mathrm{~min}^{-1}$ dry gas flow $\left(N_{2}\right), 350{ }^{\circ} \mathrm{C}$ dry gas temperature and $4.5 \mathrm{kV}$ capillary voltage. The acquired mass range was $m / z 50-800$ with an ICC smart target (instrument specific parameter) of $10^{5}$. Maximum accumulation time of the ion trap was $25 \mathrm{~ms}$. Tandem mass spectra were recorded in the auto MS/MS mode with a fragmentation amplitude of $1.00 \mathrm{~V}$ (instrument specific parameter) and an active exclusion after 3 spectra.
The UV/Vis chromatograms were screened for UV/Vis signals $>300 \mathrm{~nm}$ appearing or increasing in intensity after 1 week of reaction time (see Fig. S8-S15, ESI $\dagger$ ). If available from UV/Vis spectrophotometric analysis, wavelengths of absorbance maxima appearing in the actinic flux region (or shortly below, $\lambda_{\text {min }}=250 \mathrm{~nm}$ ) were used to screen samples from the respective reaction mixtures by HPLC-DAD-ESI-MS. Mass spectra at the retention time of the corresponding UV/Vis chromatograms were used to obtain the molecular mass of the compounds of interest. The obtained $\mathrm{m} / \mathrm{z}$ were then used in a next step to investigate structural properties of the compound by UHPLCESI-HRMS $^{2}$ analysis as described in the following section.

\section{UHPLC-HESI-HRMS ${ }^{2}$ measurements}

Ultra-high resolution tandem mass spectrometry was used to obtain structural information about products from (di-)carbonyl amine reactions. Samples were analyzed in duplicate by UHPLC-HESI-HRMS $^{2}$ using an Orbitrap mass analyzer (Q-Exactive hybrid quadrupole orbitrap mass spectrometer, Thermo Scientific, Germany) equipped with an UHPLC-System (Dionex UltiMate 3000 UHPLC system, Thermo Scientific, Germany) and a Hypersil Gold, C18, $50 \times 2.0 \mathrm{~mm}$ column with $1.9 \mu \mathrm{m}$ particle size (Thermo Scientific, Germany). Targeted MS/MS was performed for $\mathrm{m} / \mathrm{z}$ values, which were observed to contribute to the absorption $>250 \mathrm{~nm}$ from HPLC-DAD-ESI-MS/MS analysis. The injection volume was $10 \mu \mathrm{L}$ and the eluents were ultrapure water with $2 \%$ acetonitrile and $0.04 \%$ formic acid (eluent A), and acetonitrile with $2 \%$ water (eluent $\mathrm{B}$ ). The gradient of the mobile phase with a flow

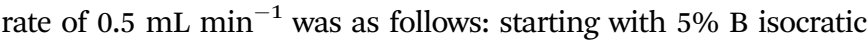
for $0.5 \mathrm{~min}$, increasing to $75 \% \mathrm{~B}$ in $2 \mathrm{~min}$, isocratic for $1 \mathrm{~min}$, increasing to $100 \% \mathrm{~B}$ in $0.5 \mathrm{~min}$, isocratic for $4 \mathrm{~min}$ and decreasing to $5 \% \mathrm{~B}$ in $0.5 \mathrm{~min}$. Mass spectrometric analyses were performed using a heated-ESI (HESI) source under the following conditions: $150{ }^{\circ} \mathrm{C}$ HESI temperature, $4 \mathrm{kV}$ spray voltage, 30 psi sheath gas flow, 20 psi auxiliary gas flow and $320{ }^{\circ} \mathrm{C}$ capillary temperature. Mass resolution was 35000 and acquired mass range was $m / z 60-500$, except for samples containing GA, where a range of $m / z$ 60-900 was used. Fragmentation energies used were generally $35 \mathrm{NCE}$ (instrument specific parameter), if not specified otherwise.

\section{Assignment of tentative structures to light-absorbing compounds}

To assess the formation of light-absorbing products in the investigated reaction mixtures, analysis results of sample aliquots taken at 0.5 and $144 \mathrm{~h}$ reaction time were compared. Differential UV/Vis analysis allowed for an evaluation of the potential of individual and mixed dicarbonyl species in the presence of selected amine species to form secondary BrC. Compound specific absorbance properties were linked to the corresponding molecular masses by HPLC-DAD-ESI-MS analysis. Finally, high mass resolution fragment ion spectra obtained by UHPLC-ESIHRMS $^{2}$ analysis in combination with mechanistic considerations were used for a tentative assignment of chemical structures to the products of interest (see Fig. 1). 


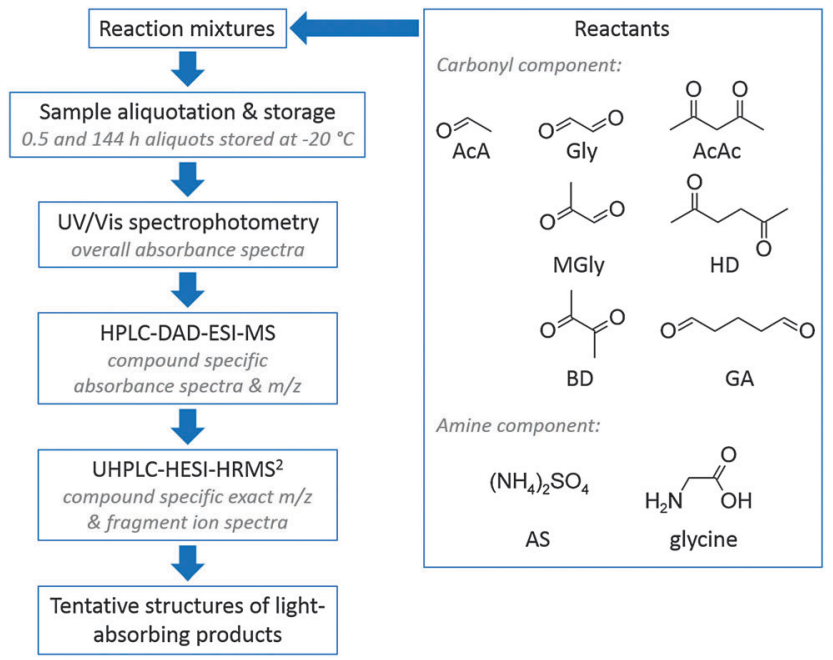

Fig. 1 Workflow for characterization and tentative structure assignment of light-absorbing compounds.

\section{Results and discussion}

\section{Changes in bulk absorption}

Fig. 2 shows absorbance spectra of the dicarbonyl amine reaction systems for $0.5 \mathrm{~h}$ and $144 \mathrm{~h}$ reaction time. Additionally, absorbance spectra of equally concentrated aqueous dicarbonyl solutions are shown for comparison. Individual UV/Vis spectra for all investigated reaction systems and dicarbonyl educts are presented in Fig. S1-S7 in the ESI. $\dagger$ Generally, an increase of the absorbance was observed for all reaction systems, indicating the formation of reaction products.

Fig. 3 shows a zoom-in of the absorbance spectra for the wavelength range with a significant actinic flux (number of incident photons) in the troposphere, i.e., wavelengths $>300 \mathrm{~nm}$. Here, 1,4- and 1,5-dicarbonyl/AS systems show distinct absorbance maxima, but also the absorbance of the other dicarbonyl/AS systems increases due to increasing concentrations of products with absorbance maxima between 250-300 nm. The development of absorbance maxima at 220 and $280 \mathrm{~nm}$, previously attributed to $1 H$-imidazole (IM) and some derivatives hereof, for 1,2-dicarbonyl/AS reactions ${ }^{16,19,21,23,25,27,29}$ is especially pronounced for MGly, while Gly and BD show no significant absorbance increase under the conditions applied in this study. It should be noted that solutions containing BD are colored slightly yellow due to the intrinsic absorption properties of BD (see Fig. 3c). 1,4- and 1,5-dicarbonyls (HD and GA) showed dark brown colors already after 30 minutes reaction time, but were colorless before mixing with AS (see Fig. 3d), indicating higher rate constants for the formation of colored products compared to 1,2- and 1,3-dicarbonyls. In Fig. 2a and 3a a broad absorption up to $\lambda \sim 550 \mathrm{~nm}$ and distinct absorption maxima at $\lambda \sim 330$ and $450 \mathrm{~nm}$ appear for HD and GA, respectively.

When glycine is supplied as the amine nucleophile component, the UV/Vis spectra of all dicarbonyls, with the exception of AcAc and GA, develop further absorbance maxima at $\lambda>250 \mathrm{~nm}$ and generally show stronger absorbance compared to reactions with AS (see Fig. 2b and 3b). Most dicarbonyls, again with the exception of AcAc, develop absorbance maxima in the actinic
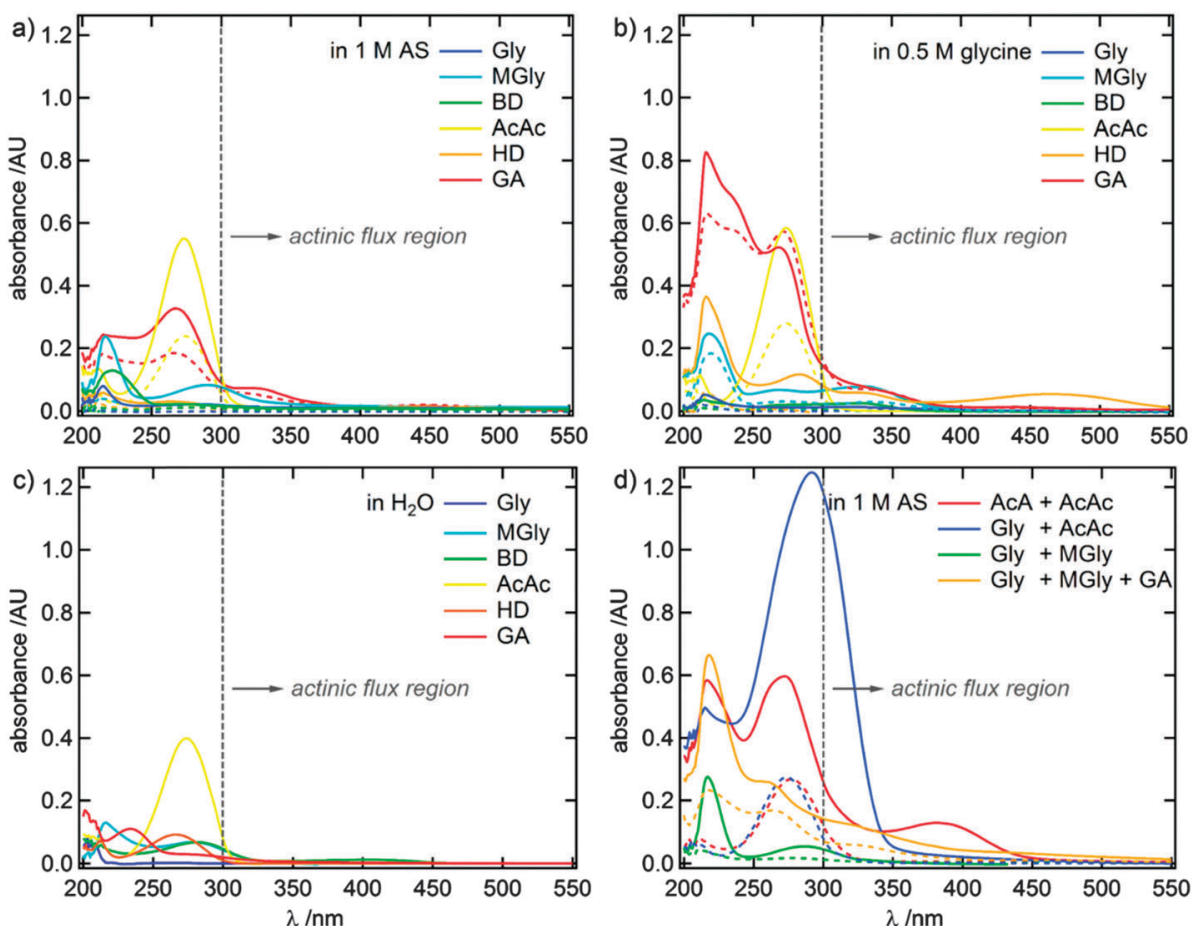

Fig. 2 UV/Vis spectra of $1 \mathrm{M}$ dicarbonyls in (a) AS (1 M), (b) glycine (0.5 M), and (c) $\mathrm{H}_{2} \mathrm{O}$; (d) UV/Vis spectra of dicarbonyl mixtures (1 M) in AS (1 M). All samples were diluted by a factor of 167 before analysis. Dashed colored lines represent spectra recorded after $0.5 \mathrm{~h}$ reaction time, solid lines are spectra recorded after $144 \mathrm{~h}$ reaction time. 

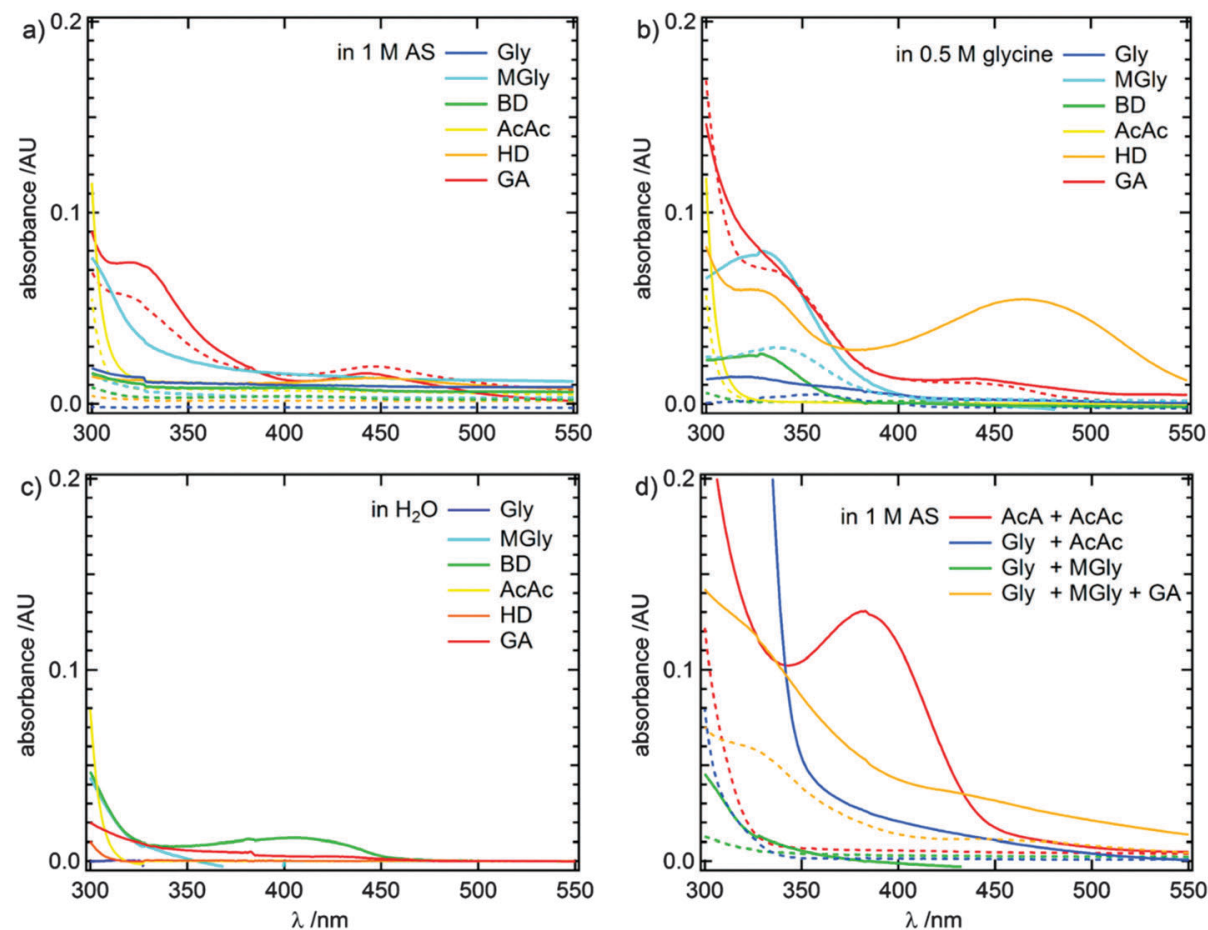

Fig. 3 Zoom in of UV/Vis spectra for the actinic flux wavelength region: $1 \mathrm{M}$ dicarbonyls in (a) AS (1 M), (b) glycine $(0.5 \mathrm{M})$, and (c) $\mathrm{H}_{2} \mathrm{O}$; (d) UV/Vis spectra of dicarbonyl mixtures (1 M) in AS (1 M). All samples were diluted by a factor of 167 before analysis. Dashed colored lines represent spectra recorded after $0.5 \mathrm{~h}$ reaction time, solid lines are spectra recorded after $144 \mathrm{~h}$ reaction time.

flux wavelength region. A common absorbance feature is visible at 320-350 $\mathrm{nm}$. The absorbance maximum in the visible wavelength range of HD is more pronounced and shifted to around $460 \mathrm{~nm}$ compared to the feature at $450 \mathrm{~nm}$ appearing in AS containing solutions.

Fig. $2 \mathrm{~d}$ and $3 \mathrm{~d}$ show UV/Vis spectra of mixed (di-)carbonyl/AS systems after $0.5 \mathrm{~h}$ and $144 \mathrm{~h}$ reaction time, respectively. The spectra of the Gly + MGly/AS system do not differ significantly from the spectra of the individual G/AS and MG/AS systems (compare Fig. S1, ESI $\dagger$ ). This result could be expected, because both dicarbonyl/AS are known to mainly produce imidazole derivatives absorbing light at $\lambda \sim 220$ and $280 \mathrm{~nm} .^{20,21,23,24,27,29,33,35,44}$ Formation of mixed imidazoles from Gly/MGly cross-reactions was observed and confirmed by mass spectrometry (see Section 3.3). The addition of GA as a third dicarbonyl to the Gly + MGly/AS system results in three absorbance maxima at 220, 260, and $320 \mathrm{~nm}$, respectively, extending into the visible wavelength range.

The Gly + AcAc/AS system shows a strong increase of absorbance at $\sim 290 \mathrm{~nm}$ leading to the most pronounced absorbance in the actinic flux region of the mixed systems investigated in this study, likely due to the activity of AcAc (see Fig. 2a), but no new maxima beyond $300 \mathrm{~nm}$ were observed. While AcA/AS and AcAc/AS do not produce species with absorbance maxima beyond $\lambda>300 \mathrm{~nm}$, the $\mathrm{AcA}+\mathrm{AcAc} / \mathrm{AS}$ spectra show a maximum at $\lambda \sim 380 \mathrm{~nm}$.

\section{Development of individual BrC chromophores}

Fig. 4 shows results of the HPLC-DAD analysis for the AcA + AcAc/AS system. In the following, this reaction system will be used to exemplarily walk the reader through the procedure to obtain structural information of potentially relevant lightabsorbing products. Corresponding plots for the other reaction systems are shown in Fig. S8-S15 in the ESI. $\dagger$

The image plots in Fig. 4 illustrate that a variety of synergistic products, i.e., products that are not formed in the individual dicarbonyl/AS systems, are formed over the course of the reaction time, some of which show absorbance properties relevant for the actinic flux wavelength region. It should be noted that the broad signal eluting from 6-8.5 min retention time (RT) with an absorbance maximum at $\lambda \sim 280 \mathrm{~nm}$ is caused by AcAc (see Fig. S11, ESI $\dagger$ ). The bottom chromatogram in Fig. 4 plots the sum

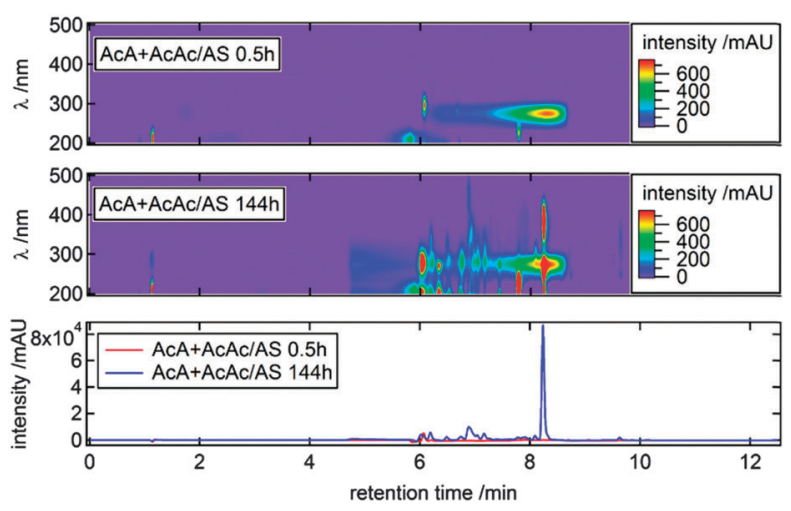

Fig. 4 HPLC-DAD analysis image plot for ACA + AcAc/AS: 0.5 (top) and $144 \mathrm{~h}$ (middle) reaction time; bottom: UV/Vis chromatogram, $\lambda=300-$ $500 \mathrm{~nm}$. The signal at $8.3 \mathrm{~min}$ RT accounts for $\sim 51 \%$ of the total absorbance in the plotted wavelength range. 


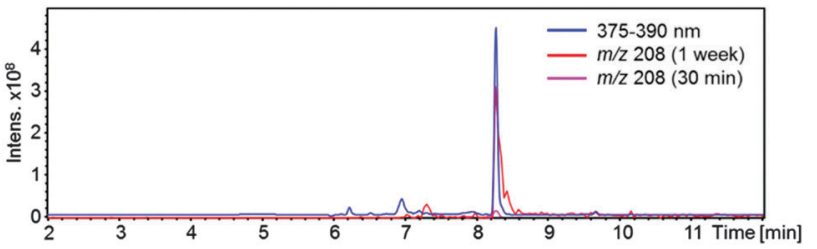

Fig. 5 UV/Vis chromatogram (375-390 nm, scale not shown) and extracted ion chromatograms (EIC) of $\mathrm{m} / \mathrm{z} 208$ at 0.5 and $144 \mathrm{~h}$ reaction time for the AcA + AcAc/AS system.

of the absorbance signals between $300 \mathrm{~nm}<\lambda<500 \mathrm{~nm}$ versus $\mathrm{RT}$, and shows that the synergistic product eluting at $8.3 \mathrm{~min}$ RT dominates the absorbance in this wavelength range. Moreover, this compound is responsible for the absorbance maximum at $\lambda \sim 380 \mathrm{~nm}$ observed using UV/Vis spectrometry for this system (see Fig. $2 \mathrm{~d}$ and $3 \mathrm{~d}$ ).

In Fig. 5 the UV/Vis chromatogram for $\lambda=375-390 \mathrm{~nm}$, corresponding to one of the absorbance maxima of the aforementioned compound, is shown together with the extracted ion chromatograms (EIC) of $m / z 208$ for $0.5 \mathrm{~h}$ and $144 \mathrm{~h}$ reaction time. The overlapping signals indicate that a compound with $\mathrm{m} / \mathrm{z} 208$ is responsible for the observed absorbance. Furthermore, it can be seen that the signal of $\mathrm{m} / \mathrm{z} 208$ develops over time.

To obtain more information about the identity and structure of this reaction product UHPLC-HESI-HRMS ${ }^{2}$ experiments were performed. Fig. 6 shows an exemplary high-resolution $\mathrm{MS}^{2}$ spectrum for the compound with $\mathrm{m} / \mathrm{z} 208$ identified to be responsible for the observed absorption at $\lambda \sim 380 \mathrm{~nm}$ in the AcA + AcAc/AS system. The high mass resolution of the Orbitrap mass analyzer $(R>35.000)$ allows for the assignment of sum formulas to the observed signals, e.g., the ion with the exact $m / z$ of 208.1337 corresponds to a $\mathrm{C}_{12} \mathrm{H}_{18} \mathrm{O}_{2} \mathrm{~N}$ compound. According to the sum formulas, the nitrogen $(\mathrm{N})$ atom remains present in all fragment ions, indicating its position within a cyclic, potentially aromatic, structure. The protonated molecular ion being the base peak in the $\mathrm{MS}^{2}$ spectrum indicates its high stability and further suggests aromatic or aromatic-like structural properties of $m / z$ 208.1337. In addition, the fragment ion signals suggest the presence of three exocyclic methyl groups as well as two exocyclic acetyl groups in the molecule. In summary, the UHPLC-HESI-HRMS $^{2}$ analysis of the AcA + AcAc/AS reaction system indicates that a six membered ring nitrogen heterocycle with three methyl and two acetyl substituents is responsible for the majority of the observed absorbance of this reaction system.

High resolution $\mathrm{MS}^{2}$ spectra for potentially relevant products formed in the other reaction systems can be found in Fig. S16-S32 in the ESI. $\dagger$ It should be noted, that for GA/AS no $\mathrm{MS}^{2}$ spectra of relevant compounds are presented, because the polymer signals observed using the HPLC-DAD-ESI-MS ${ }^{2}$ were absent in the UHPLC-HESI-HRMS ${ }^{2}$ analyses.

\section{Tentative structures of the observed light-absorbing products}

The identification of individual light-absorbing species in mixtures mimicking atmospheric aerosols producing $\mathrm{BrC}$ has been recognized as a challenging task. ${ }^{54}$ As reactions between six different dicarbonyl species and two different amine species, totaling to sixteen reaction systems, were investigated in this study, standard C18 reversed phase columns were used to achieve reasonable product separations for all reaction systems. Based on the information obtained using the analyses outlined above and mechanistic considerations, tentative structures for selected products are proposed. Again, this shall be discussed exemplarily for the product with the exact $\mathrm{m} / \mathrm{z} 208.1337$ observed in the AcA + AcAc/AS system.

The AcA + AcAc/AS system represents a well-known reaction system in organic chemistry: 1,3-dicarbonyls react with aldehydes in the presence of ammonia to produce 1,4-dihydropyridines following the reaction described by Hantzsch in the late 19th century. ${ }^{55}$ Fig. 7 illustrates the current view of the corresponding reaction mechanism. Briefly, ammonia nucleophilically attacks at one of the carbonyl carbon atoms of acetylacetone (I), yielding the enamine (III) after dehydration of hemiaminal (II). Enamine III then reacts with intermediate VI, which was formed by nucleophilic attack of the enolic form of AcAc (Ia) at the AcA (IV) carbonyl carbon atom followed by a dehydration step of the intermediate aldol addition product V. The reaction of III and VI yields an imine (VII), which causes intramolecular cyclization to intermediate VIII, which is followed by another dehydration step to yield the dihydropyridine product IX. The corresponding

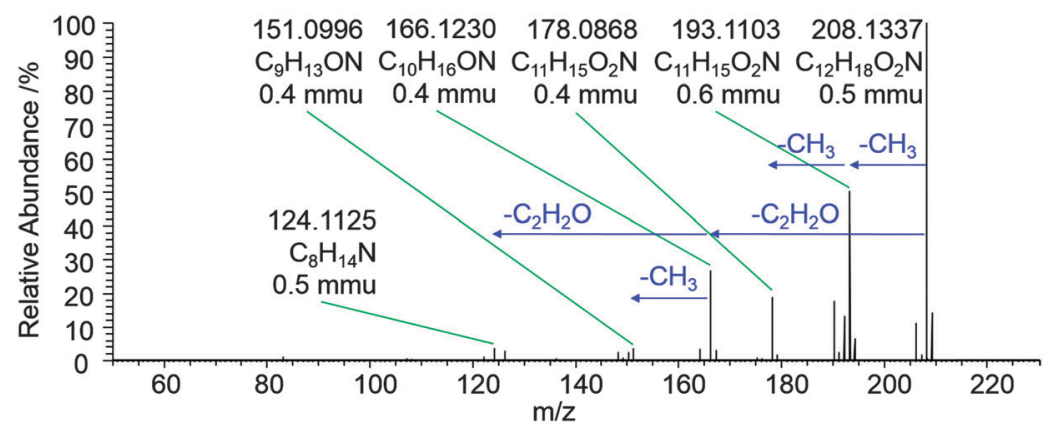

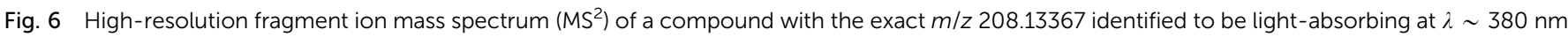

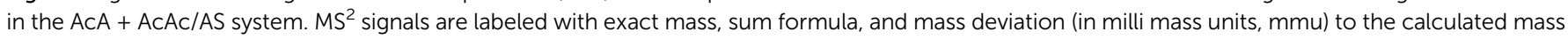
of the respective sum formula. Blue arrows indicate proposed neutral losses. 

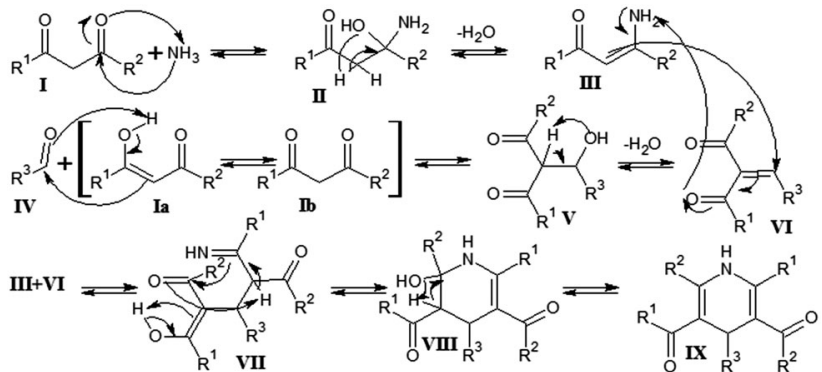

Fig. 7 Mechanism of the Hantzsch 1,4-dihydropyridine synthesis. ${ }^{55}$ In the $A c A+A c A c / A S$ system $R^{1}, R^{2}$, and $R^{3}$ are methyl groups. Product identity was confirmed to be 3,5-diacetyl-2,4,6-trimethyl-1,4-dihydropyridine by reference compound analysis (Fig. S34, ESI $\dagger$ ).

product is 3,5-diacetyl-2,4,6-trimethyl-1,4-dihydropyridine, termed dihydropyridine product hereafter. The dihydropyridine product has an absorption maximum at $375 \mathrm{~nm}\left(\varepsilon=6300 \mathrm{~L} \mathrm{~mol}^{-1} \mathrm{~cm}^{-1}\right){ }^{56}$ which is in good agreement with the observed absorption behavior in the reaction mixture. Further, it has a nominal mass of $207 \mathrm{Da}$ and an exact mass of $208.13321 \mathrm{Da}$, when protonated. Also, the fragment ions observed in the $\mathrm{MS}^{2}$ spectrum of $\mathrm{m} / \mathrm{z} 208.1337$ (Fig. 6) are in agreement with the acetyl and methyl substituents of the dihydropyridine product.

For a few of the tentatively identified structures of lightabsorbing products summarized in Table 2, reference compounds were commercially available, i.e., for $1 H$-imidazole (IM, Gly/AS system), $1 H$-imidazole-2-carbaldehyde (IC, Gly/AS system), 3-acetyl2,4,6,-trimethylpyridine (pyridine product, AcAc/AS system), and 3,5-diacetyl-2,4,6-trimethyl-1,4-dihydropyridine (dihydropyridine product, AcA + AcAc/AS system). For these cases, the identity of the products was confirmed by UHPLC-HESI-HRMS ${ }^{2}$ retention time and $\mathrm{MS}^{2}$ spectra (see Fig. S33-S36 in the ESI $\dagger$ ).

1,2-Dicarbonyl/AS and /glycine reactions yielded the expected products described previously. ${ }^{21,23,24,27,44}$ The imidazole products 1, 2, and 3 have been identified in the literature as products contributing to the observed browning of Gly/AS and /glycine systems..$^{21,23,27,33,35,44}$ The presence of $\mathbf{1}$ and $\mathbf{2}$ in the investigated Gly/AS mixtures was confirmed by reference compounds. Similarly, products in the MGly/AS and/glycine systems include imidazole derivatives, e.g., 4-methyl- $1 H$-imidazole (4, MIM) and 2-acetyl-4-methyl- $1 H$-imidazole (5, AMIM) ${ }^{24}$ The acetyl group of AMIM can undergo further aldol condensation with an MGly monomer yielding product 6 in Table 2. For BD/AS, no product structures are proposed, because the absorption spectrum actually shifts towards shorter wavelengths compared to $\mathrm{BD}$ in $\mathrm{H}_{2} \mathrm{O}$ (see Fig. 2a and c). In the $\mathrm{BD} /$ glycine system an absorption feature at $320 \mathrm{~nm}$ develops, to which no tentative structure could be assigned because no plausible mechanism could yet be proposed explaining the observed molecular formula. For the same reason, no structure was assigned to the product with $m / z 153\left(\mathrm{C}_{8} \mathrm{H}_{13} \mathrm{ON}_{2}\right)$ in the Gly + MGly + GA/AS system (product 22). While the identity of product 10 in the AcAc/AS system was confirmed by analysis of a 3-acetyl-2,4,6trimethyl-pyridine reference, product $\mathbf{1 1}$ in the AcAc/glycine system appears to be an open chain condensation product, which is stabilized through an intramolecular hydrogen bond.
Products 12 and 13, tentatively identified in the HD/AS system, consist of coupled 2,5-dimethylpyrrole units, which are also likely to be found in the $\mathrm{HD} /$ glycine reaction systems (product 15). Spectroscopic information for product 14 suggests the presence of a pyrrole unit in the molecule, while no dipyrromethene is formed (absence of absorbance at $450 \mathrm{~nm}$ ). Besides the oligomeric species observable in the broad UV/Vis signal (see Fig. S13, ESI $\dagger$ ) of GA/AS and GA/glycine systems, for which no product structures are proposed due to missing signals in the HRMS analyses, pyridine (product 16) and its $\mathrm{N}$-substituted acetic acid derivative (product 17) could be identified. Also in mixed dicarbonyl experiments, the presence of nitrogen heterocycles could be confirmed, e.g., 3,5-diacetyl-2,4,6-trimethyl-1,4dihydropyridine (product 18) in the AcA + AcAc/AS system via analysis of a reference compound. While Gly + AcAc/AS yielded a substituted dihydropyrrole (product 19), the Gly + MGly/AS system yielded the expected mixed imidazole derivatives (products 20 and 21) in addition to those formed in Gly/AS and MGly/AS reactions.

\section{The chemistry of the formation of nitrogen heterocycles in aqueous aerosol mimics}

For nearly all studied reaction systems, we tentatively identify nitrogen containing heterocycles as common structural motifs of BrC chromophores. These structures are based on reactions involving the nucleophilic attack of the respective amine species at reactive carbonyl sites followed by intra- or intermolecular cyclization and in several cases dehydration to the corresponding heteroaromatic ring systems as detailed below. Another typical reaction under the present conditions is the acid catalyzed aldol reaction, which leads to $\mathrm{C}-\mathrm{C}$-bond formation. ${ }^{18,20,46,49}$

Glyoxal in aqueous solution is characterized by a fast equilibrium between hydrated and non-hydrated aldehyde functions, providing reactive and sterically accessible carbonyl groups for the nucleophilic attack of primary amines like ammonia or amino acids, while self-aldol reactions are slow due to unfavorable keto enol tautomerism (lacking + I effect of an additional alkyl group). Thus the main products in the corresponding reaction systems involve imidazole ring structures, i.e., five membered aromatic rings with two nitrogen atoms. Methylglyoxal can also form imidazole derivatives via the imine pathway as demonstrated here and in the literature. ${ }^{24,57}$ The IC analog AMIM retains some reactivity towards further aldol condensation compared to IC and thus also higher molecular weight products can be observed for MGly (product 6). The proposed structure features a substitution of the hydrogen in 1-position of the imidazole ring by another MGly monomer, which has also been described for glyoxal before. ${ }^{27}$ For the diketone BD, no imidazole products could be observed, potentially due to the trimerization of BD by formation of cyclic acetals. ${ }^{58,59}$ Consequently, BD/AS did not yield products absorbing at wavelengths $>250 \mathrm{~nm}$ within the timescale and conditions of our experiments.

AcAc/AS and/glycine constitute 1,3-dicarbonyl/amine reaction systems. Acetylacetone, like all $\beta$-diketones, favors the enol-tautomer since the possible hydrogen bond between the two oxygen 
Table 2 Summary of tentatively identified product structures and corresponding UV/Vis spectroscopic, chromatographic and mass spectrometric data. Mass spectrometric signals with high abundance in fragment ion spectra are given in bold font, signals with $<10 \%$ relative intensity are given in italic font, respectively

\begin{tabular}{|c|c|c|c|c|c|c|}
\hline \# & Reaction system & Tentative structure & $\begin{array}{l}\mathrm{RT} / \mathrm{min} \text { (HPLC/ } \\
\text { UHPLC) }\end{array}$ & $\begin{array}{l}\text { Exact } m / z \text { of }[\mathrm{M}+\mathrm{H}]^{+} \\
\text {(sum formula) }\end{array}$ & Fragment ion $m / z$ & $\lambda_{\max } / \mathrm{nm}$ \\
\hline 1 & ${ }^{a}$ Gly/AS & $\underset{N}{H}$ & $1.2 / 0.34$ & $69.0455\left(\mathrm{C}_{3} \mathrm{H}_{5} \mathrm{~N}_{2}\right)$ & - & 220 \\
\hline 2 & ${ }^{a}$ Gly/AS & $\mathrm{H}$ & $1.1 / 0.33$ & $115.0506\left(\mathrm{C}_{4} \mathrm{H}_{7} \mathrm{O}_{2} \mathrm{~N}_{2}\right)$ & $\mathbf{9 7 . 0 4}, 69.05$ & 260 \\
\hline 3 & Gly/glycine & & $1.1 / 0.34$ & 185.0555 $\left(\mathrm{C}_{7} \mathrm{H}_{9} \mathrm{O}_{4} \mathrm{~N}_{2}\right)$ & $139.05,111.06$ & 220,330 \\
\hline 4 & MGly/AS & $\mathrm{H}$ & $1.2 / 0.35$ & 83.0612 $\left(\mathrm{C}_{4} \mathrm{H}_{7} \mathrm{~N}_{2}\right)$ & 56.05 & 220 \\
\hline 5 & MGly/AS & & $3.9 / 0.64$ & $125.0711\left(\mathrm{C}_{6} \mathrm{H}_{9} \mathrm{ON}_{2}\right)$ & 83.06 & 280 \\
\hline 6 & MGly/AS & & $7.7 / 1.75$ & $251.1027\left(\mathrm{C}_{12} \mathrm{H}_{15} \mathrm{O}_{4} \mathrm{~N}_{2}\right)$ & $\begin{array}{l}233.09,209.09,191.08 \\
167.08,139.09\end{array}$ & 285 \\
\hline 7 & MGly/glycine & & $6.8 / 1.56$ & $184.0605\left(\mathrm{C}_{8} \mathrm{H}_{10} \mathrm{O}_{4} \mathrm{~N}\right)$ & $\begin{array}{l}\text { 156.07, 138.05, 128.07, } \\
\text { 110.06, 82.07 }\end{array}$ & 265,330 \\
\hline 8 & $\mathrm{BD} / \mathrm{AS}$ & 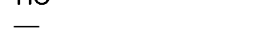 & $7.8 / 1.80$ & $112.0762\left(\mathrm{C}_{6} \mathrm{H}_{10} \mathrm{ON}\right)$ & 70.07 & \\
\hline 9 & $\mathrm{BD} /$ glycine & - & $6.1 / 1.48$ & $254.1020\left(\mathrm{C}_{12} \mathrm{H}_{16} \mathrm{O}_{5} \mathrm{~N}\right)$ & $236.09,208.10,190.09,161.06$ & 215,320 \\
\hline 10 & ${ }^{a} \mathrm{AcAc} / \mathrm{AS}$ & & $6.3 / 0.64$ & $164.1065\left(\mathrm{C}_{10} \mathrm{H}_{14} \mathrm{ON}\right)$ & $136.08,122.10,121.09$ & 215,270 \\
\hline 11 & AcAc/glycine & & $7.3 / 1.34$ & $158.0817\left(\mathrm{C}_{7} \mathrm{H}_{12} \mathrm{O}_{3} \mathrm{~N}\right)$ & $116.07, \mathbf{1 1 2 . 0 8}, 94.07,70.07$ & 290 \\
\hline 12 & $\mathrm{HD} / \mathrm{AS}$ & $\mathrm{H}$ & $7.6 / 1.76$ & $187.1232\left(\mathrm{C}_{12} \mathrm{H}_{15} \mathrm{~N}_{2}\right)$ & 146.10 & 450 \\
\hline 13 & $\mathrm{HD} / \mathrm{AS}$ & & $7.7 / 1.83$ & $201.1388\left(\mathrm{C}_{13} \mathrm{H}_{17} \mathrm{~N}_{2}\right)$ & 160.11 & 350,450 \\
\hline 14 & HD/glycine & & $6.9 / 1.55$ & 248.1277 $\left(\mathrm{C}_{14} \mathrm{H}_{18} \mathrm{O}_{3} \mathrm{~N}\right)$ & $230.12,202.12,184.11,173.10$ & 220,330 \\
\hline 15 & HD/glycine & & $7.1 / 1.57$ & $317.1497\left(\mathrm{C}_{17} \mathrm{H}_{21} \mathrm{O}_{4} \mathrm{~N}_{2}\right)$ & $216.10,100.04,72.05$ & 450 \\
\hline 16 & GA/AS & & $0.9 / 0.36$ & $80.0501\left(\mathrm{C}_{5} \mathrm{H}_{6} \mathrm{~N}\right)$ & - & 240 \\
\hline
\end{tabular}


Table 2 (continued)

\begin{tabular}{|c|c|c|c|c|c|c|}
\hline \# & Reaction system & Tentative structure & $\begin{array}{l}\mathrm{RT} / \mathrm{min} \text { (HPLC/ } \\
\text { UHPLC) }\end{array}$ & $\begin{array}{l}\text { Exact } m / z \text { of }[\mathrm{M}+\mathrm{H}]^{+} \\
\text {(sum formula) }\end{array}$ & Fragment ion $\mathrm{m} / \mathrm{z}$ & $\lambda_{\max } / \mathrm{nm}$ \\
\hline 17 & GA/glycine & & $1.1 / 0.36$ & $138.0548\left(\mathrm{C}_{7} \mathrm{H}_{8} \mathrm{O}_{2} \mathrm{~N}\right)$ & 93.08 & 240,260 \\
\hline 18 & ${ }^{a} \mathrm{AcA}+\mathrm{AcAc} / \mathrm{AS}$ & & $8.3 / 1.78$ & $208.1338\left(\mathrm{C}_{12} \mathrm{H}_{18} \mathrm{O}_{2} \mathrm{~N}\right)$ & $\begin{array}{l}\text { 193.11, 190.12, } 178.09 \\
166.12,151.10,124.11\end{array}$ & 255,385 \\
\hline 19 & Gly + AcAc/AS & & $6.7 / 1.51$ & $140.0709\left(\mathrm{C}_{7} \mathrm{H}_{10} \mathrm{O}_{2} \mathrm{~N}\right)$ & 112.08, 98.06, 70.07 & 280 \\
\hline 20 & Gly + MGly/AS & $\mathrm{H}$ & $1.4 / 0.37$ & $111.0558\left(\mathrm{C}_{5} \mathrm{H}_{7} \mathrm{ON}_{2}\right)$ & $95.06,83.06$ & 265 \\
\hline 21 & Gly + MGly/AS & $\mathrm{H}$ & $2.5 / 0.56$ & $111.0558\left(\mathrm{C}_{5} \mathrm{H}_{7} \mathrm{ON}_{2}\right)$ & $83.06,69.05$ & 265 \\
\hline 22 & Gly + MGly + GA/AS & - & $3.7 / 0.61$ & 153.1029 $\left(\mathrm{C}_{8} \mathrm{H}_{13} \mathrm{ON}_{2}\right)$ & $109.08,96.07$ & 220 \\
\hline
\end{tabular}

atoms forms a six membered ring. Thus, 1,3-dicarbonyls are very reactive and act as nucleophiles in aldol reactions. After reaction with ammonia to form the enaminone, this more nucleophilic species can react with another molecule of AcAc in an aldol reaction. The resulting product can undergo a cyclocondensation to form the corresponding pyridine (product 10). In contrast, neutral pyridine cannot form when glycine is used as the amine component and the reaction stops at the simple enamine structure (product 11). Furthermore, the enol tautomer of the keto group is favored because it enables the $\mathrm{H}$-bond with the nitrogen lone pair.

Reaction systems involving the 1,4- and 1,5-dicarbonyls HD and GA, respectively, show a fast production of $\mathrm{BrC}$ species. The corresponding samples showed strong colors already within the first hour of reaction time, for GA/glycine first colored products were actually visible within seconds. Thus, also the baseline absorbance (signals after $0.5 \mathrm{~h}$ of reaction) was relatively high (see Fig. 2 and 3). The chemistry of HD allows the formation of nitrogen heterocycles, i.e., 2,5-dimethylpyrrole following the Paal-Knorr synthesis. ${ }^{60-62}$ These pyrroles are very sensitive towards oxidation at the methyl groups which enables coupling reactions and the formation of dimers (products 12, 13, and 15). These products show a larger conjugated $\pi$-electron system than the simple pyrrole frameworks, which leads to a bathochromic shift of the absorbance maximum to the visible region around $450 \mathrm{~nm} .{ }^{63}$ Identification of specific BrC chromophores derived from GA has proven difficult due to the pronounced and diverse oligomerization reactions of GA. ${ }^{64}$ HPLC-DAD-ESIMS data suggests a partial conversion of GA oligomer carbonyl functions into imines as indicated by odd $\mathrm{m} / \mathrm{z}$ for some of the observed oligomer signals and thus an odd number of $\mathrm{N}$-atoms in the ions (data not shown). In the reaction of GA with AS and glycine, we propose the formation of ( $N$-substituted) pyridine, i.e., products 16 and $\mathbf{1 7}$ in Table 2, via cyclization and formation of an aromatic system after imine formation.

Recently, browning of laboratory generated SOA from ozonolysis reactions of biogenic and anthropogenic VOCs collected on filters and exposed to ammonia gas was observed ${ }^{28}$ and a second generation oxidation product in the ozonolysis of limonene was confirmed as a potent precursor for the observed browning of limonene SOA. ${ }^{31}$ Nguyen et al. proposed that ketolimononaldehyde first reacts as an 1,4-dicarbonyl according to Paal and Knorr forming a pyrrole derivative, which is then further oxidized and dehydrated yielding an exocyclic conjugated double bond system, and finally dimerizes in a pyrrole coupling reaction. The proposed product has the sum formula $\mathrm{C}_{18} \mathrm{H}_{20} \mathrm{~N}_{2} \mathrm{O}_{2}$ (10 double bond equivalents, DBE) and a strong absorption maximum at $\lambda \sim 500 \mathrm{~nm}$. Interestingly, the corresponding first generation ozonolysis product of limonene, i.e., the 1,6-dicarbonyl limononaldehyde, did not produce a similarly absorbing product, indicating that 1,6-ketoaldehydes are not able to produce relevant chromophores. Similarly, the first generation ozonolysis product of $\alpha$-pinene, pinonaldehyde, a 1,6-dicarbonyl, was unable to produce BrC. ${ }^{31}$ The sterically unhindered 1,4- and 1,5-dicarbonyl components studied in this work produced $\mathrm{BrC}$ species efficiently, i.e., within seconds (GA/glycine) to minutes. Tentative assignment of structures to GA derived $\mathrm{BrC}$ chromophores besides pyridine formed via a self-cyclization of an GA imine (products 16 and 17) was not possible as outlined above. In accordance to observations of limonene SOA browning, pyrroles and dipyrromethenes, i.e., coupled pyrrole systems, were observed in the HD amine reactions. Absorbance maxima of the dipyrromethenes proposed here also 
match the absorbance features observed for the limonene SOA BrC chromophores reported by Nguyen et al., ${ }^{31}$ confirming the relevance of terpene derived 1,4-dicarbonyls as BrC precursors.

Mixed (di-)carbonyl/amine systems were investigated for synergistic cross-reaction products. While cross-reaction products in the Gly + MGly/AS system (products 20 and 21) did not differ significantly from the respective products in the individual dicarbonyl/AS systems regarding their absorbance properties (see Fig. 2d and 3d), other mixed systems developed crossreaction products with absorbance maxima shifted towards the actinic flux region. In the AcA + AcAc/AS system, for which the individual (di-)carbonyl/AS systems did not develop any relevant products, the addition of a second carbonyl component (e.g., acetaldehyde) leads to the formation of a stable synergistic product, i.e., a substituted dihydropyridine (product 18). This product now possesses a significant light absorption near visible wavelengths $(\lambda \sim 380 \mathrm{~nm}) .{ }^{55,56}$ Also in the Gly + AcAc/AS system we observe a shift of the absorbance feature at $\lambda=260$ and $280 \mathrm{~nm}$ for Gly/AS and AcAc/AS, respectively, to around $290 \mathrm{~nm}$, indicating a cross-reaction product with slightly different absorption spectrum. While the GA/AS system yields a range of imine substituted aldol condensation oligomers as mentioned above, the addition of Gly and MGly to the system results in the formation of several distinct species (see Fig. S15b, ESI $\dagger$ ). These results indicate that in complex mixtures of carbonyl and amine species, which are likely to be encountered in atmospheric particulates, competitive and synergistic cross-reactions can influence the structures, and thus absorption properties, of BrC chromophores formed via the imine pathway. Further atmospherically relevant mixed dicarbonyl systems should therefore be investigated in follow-up studies.

\section{Conclusions}

We propose that nitrogen heterocycles, such as imidazoles, pyrroles, (dihydro-)pyridines, and dipyrromethenes are common BrC chromophores produced in dicarbonyl amine reactions via the imine pathway. We have demonstrated that the formation of secondary BrC involves a variety of organic chemical reactions depending on the individual reaction partners as illustrated in Fig. 8. The resulting complexity of BrC products may further be enhanced by aerosol hydration cycles, e.g., upon cloud processing, because the reactivity of certain dicarbonyls may be greatly enhanced under drying conditions. ${ }^{24,29}$ Cross-reactions may result in the formation of a high number of individual compounds, some of which may be strong absorbers present in small amounts, presenting a severe analytical challenge for the identification and quantification of relevant secondary BrC marker compounds in ambient aerosols.

Notably, BrC chromophores are subject to further photochemistry due their ability to absorb photons in the actinic flux region. This gives rise to photodegradation (photobleaching), but also to photosensitization reactions. Brown carbon chromophores, in addition to influencing aerosol optical properties, thus can also contribute to heterogeneous radical chemistry, promoting aerosol particle growth via enhanced uptake of VOCs. ${ }^{38}$

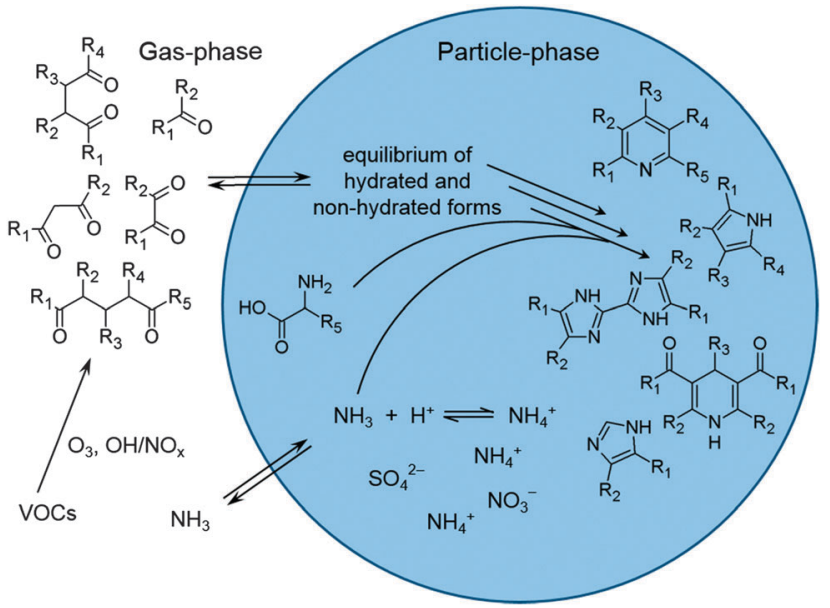

Fig. 8 Schematic overview of secondary $\mathrm{BrC}$ and potential photosensitizer formation in the aqueous aerosol phase by (di-)carbonyls and amines, yielding (synergistic) nitrogen containing heterocyclic compounds.

Moreover, we have observed synergistic cross-reactions between different dicarbonyls in amine containing solutions. While some (di-)carbonyl/amine systems do not allow the formation of thermodynamically stable conjugated or even heteroaromatic systems, such compounds can be produced when further carbonyl components with a suitable functionalization pattern are present. The synergistic products can significantly enhance the absorption in the actinic flux region, sometimes even extending to visible wavelengths, as observed for the addition of acetaldehyde to the acetylacetone/AS system and the addition of acetylacetone to the glyoxal/AS system. Further studies are necessary to identify potentially important absorbers produced by such cross-reactions in atmospherically relevant dicarbonyl mixtures.

\section{Acknowledgements}

C. J. K. acknowledges funding by the German Research Foundation (DFG; Grant no. KA 4008/1-1). C. J. K. acknowledges stimulating exchange with and infrastructural support by Ulrich Pöschl (Max Planck Institute for Chemistry). The authors thank Dirk Dienhart for technical support.

\section{References}

1 M. O. Andreae and A. Gelencser, Atmos. Chem. Phys., 2006, 6, 3131-3148.

2 A. Laskin, J. Laskin and S. A. Nizkorodov, Chem. Rev., 2015, 115, 4335-4382.

3 S. Solomon, D. Qin, M. Manning, Z. Chen, M. Marquis, K. B. Averyt, M. Tignor and H. L. Miller, IPCC Fourth Assessment Report: Climate Change 2007, Cambridge University Press, Cambridge, United Kingdom and New York, NY, USA, 2007.

4 T. F. Stocker, D. Qin, G.-K. Plattner, M. Tignor, S. K. Allen, J. Boschung, A. Nauels, Y. Xia, V. Bex and P. M. Midgley, IPCC Fifth Assessment Report: Climate Change 2013, Cambridge 
University Press Cambridge, United Kingdom and New York, NY, USA, 2013, p. 1535.

5 U. Pöschl, Anal. Bioanal. Chem., 2003, 375, 30-32.

6 U. Poschl, Angew. Chem., Int. Ed., 2005, 44, 7520-7540.

7 D. A. Lack, R. Bahreni, J. M. Langridge, J. B. Gilman and A. M. Middlebrook, Atmos. Chem. Phys., 2013, 13, 2415-2422.

8 T. C. Bond and R. W. Bergstrom, Aerosol Sci. Technol., 2006, 40, 27-67.

9 Y. Feng, V. Ramanathan and V. R. Kotamarthi, Atmos. Chem. Phys., 2013, 13, 8607-8621.

10 G. X. Lin, J. E. Penner, M. G. Flanner, S. Sillman, L. Xu and C. Zhou, J. Geophys. Res.: Atmos., 2014, 119, 7453-7476.

11 T. Moise, J. M. Flores and Y. Rudich, Chem. Rev., 2015, 115, 4400-4439.

12 X. L. Zhang, Y. H. Lin, J. D. Surratt and R. J. Weber, Environ. Sci. Technol., 2013, 47, 3685-3693.

13 J. W. Lu, J. M. Flores, A. Lavi, A. Abo-Riziq and Y. Rudich, Phys. Chem. Chem. Phys., 2011, 13, 6484-6492.

14 M. A. J. Harrison, S. Barra, D. Borghesi, D. Vione, C. Arsene and R. Iulian Olariu, Atmos. Environ., 2005, 39, 231-248.

15 P. Lin, J. Liu, J. E. Shilling, S. M. Kathmann, J. Laskin and A. Laskin, Phys. Chem. Chem. Phys., 2015, 17, 23312-23325.

16 E. L. Shapiro, J. Szprengiel, N. Sareen, C. N. Jen, M. R. Giordano and V. F. McNeill, Atmos. Chem. Phys., 2009, 9, 2289-2300.

17 D. L. Bones, D. K. Henricksen, S. A. Mang, M. Gonsior, A. P. Bateman, T. B. Nguyen, W. J. Cooper and S. A. Nizkorodov, J. Geophys. Res.: Atmos., 2010, 115, 14.

18 B. Noziere and W. Esteve, Atmos. Environ., 2007, 41, 1150-1163.

19 D. O. De Haan, M. A. Tolbert and J. L. Jimenez, Geophys. Res. Lett., 2009, 36, 5.

20 B. Noziere, P. Dziedzic and A. Cordova, J. Phys. Chem. A, 2009, 113, 231-237.

21 D. O. De Haan, A. L. Corrigan, K. W. Smith, D. R. Stroik, J. J. Turley, F. E. Lee, M. A. Tolbert, J. L. Jimenez, K. E. Cordova and G. R. Ferrell, Environ. Sci. Technol., 2009, 43, 2818-2824.

22 J. Laskin, A. Laskin, P. J. Roach, G. W. Slysz, G. A. Anderson, S. A. Nizkorodov, D. L. Bones and L. Q. Nguyen, Anal. Chem., 2010, 82, 2048-2058.

23 G. Yu, A. R. Bayer, M. M. Galloway, K. J. Korshavn, C. G. Fry and F. N. Keutsch, Environ. Sci. Technol., 2011, 45, 6336-6342.

24 D. O. De Haan, L. N. Hawkins, J. A. Kononenko, J. J. Turley, A. L. Corrigan, M. A. Tolbert and J. L. Jimenez, Environ. Sci. Technol., 2011, 45, 984-991.

25 J. Kua, H. E. Krizner and D. O. De Haan, J. Phys. Chem. A, 2011, 115, 1667-1675.

26 M. Trainic, A. A. Riziq, A. Lavi, J. M. Flores and Y. Rudich, Atmos. Chem. Phys., 2011, 11, 9697-9707.

27 C. J. Kampf, R. Jakob and T. Hoffmann, Atmos. Chem. Phys., 2012, 12, 6323-6333.

28 K. M. Updyke, T. B. Nguyen and S. A. Nizkorodov, Atmos. Environ., 2012, 63, 22-31.

29 K. J. Zarzana, D. O. De Haan, M. A. Freedman, C. A. Hasenkopf and M. A. Tolbert, Environ. Sci. Technol., 2012, 46, 4845-4851.
30 A. K. Y. Lee, R. Zhao, R. Li, J. Liggio, S. M. Li and J. P. D. Abbatt, Environ. Sci. Technol., 2013, 47, 12819-12826.

31 T. B. Nguyen, A. Laskin, J. Laskin and S. A. Nizkorodov, Faraday Discuss., 2013, 165, 473-494.

32 J. Laskin, A. Laskin, S. A. Nizkorodov, P. Roach, P. Eckert, M. K. Gilles, B. B. Wang, H. J. Lee and Q. C. Hu, Environ. Sci. Technol., 2014, 48, 12047-12055.

33 M. H. Powelson, B. M. Espelien, L. N. Hawkins, M. M. Galloway and D. O. De Haan, Environ. Sci. Technol., 2014, 48, 985-993.

34 J. M. Flores, R. A. Washenfelder, G. Adler, H. J. Lee, L. Segev, J. Laskin, A. Laskin, S. A. Nizkorodov, S. S. Brown and Y. Rudich, Phys. Chem. Chem. Phys., 2014, 16, 10629-10642.

35 A. Maxut, B. Noziere, B. Fenet and H. Mechakra, Phys. Chem. Chem. Phys., 2015, 17, 20416-20424.

36 J. F. Hamilton, M. T. Baeza-Romero, E. Finessi, A. R. Rickard, R. M. Healy, S. Peppe, T. J. Adams, M. J. S. Daniels, S. M. Ball, I. C. A. Goodall, P. S. Monks, E. Borras and A. Munoz, Faraday Discuss., 2013, 165, 447-472.

37 T. C. Bond, C. Zarzycki, M. G. Flanner and D. M. Koch, Atmos. Chem. Phys., 2011, 11, 1505-1525.

38 S. Rossignol, K. Z. Aregahegn, L. Tinel, L. Fine, B. Noziere and C. George, Environ. Sci. Technol., 2014, 48, 3218-3227.

39 T.-M. Fu, D. J. Jacob, F. Wittrock, J. P. Burrows, M. Vrekoussis and D. K. Henze, J. Geophys. Res.: Atmos., 2008, 113, D15303.

40 S. Myriokefalitakis, M. Vrekoussis, K. Tsigaridis, F. Wittrock, A. Richter, C. Bruhl, R. Volkamer, J. P. Burrows and M. Kanakidou, Atmos. Chem. Phys., 2008, 8, 4965-4981.

41 J. H. Kroll, N. L. Ng, S. M. Murphy, V. Varutbangkul, R. C. Flagan and J. H. Seinfeld, J. Geophys. Res.: Atmos., 2005, 110, D23207.

42 A. L. Corrigan, S. W. Hanley and D. O. De Haan, Environ. Sci. Technol., 2008, 42, 4428-4433.

43 H. S. S. Ip, X. H. H. Huang and J. Z. Yu, Geophys. Res. Lett., 2009, 36, L01802.

44 M. M. Galloway, P. S. Chhabra, A. W. H. Chan, J. D. Surratt, R. C. Flagan, J. H. Seinfeld and F. N. Keutsch, Atmos. Chem. Phys., 2009, 9, 3331-3345.

45 C. J. Kampf, E. M. Waxman, J. G. Slowik, J. Dommen, L. Pfaffenberger, A. P. Praplan, A. S. H. Prévôt, U. Baltensperger, T. Hoffmann and R. Volkamer, Environ. Sci. Technol., 2013, 47, 4236-4244.

46 B. Noziere and A. Cordova, J. Phys. Chem. A, 2008, 112, 2827-2837.

47 B. Noziere, P. Dziedzic and A. Cordova, Phys. Chem. Chem. Phys., 2010, 12, 3864-3872.

48 W. Esteve and B. Noziere, J. Phys. Chem. A, 2005, 109, 10920-10928.

49 J. Liggio, S. M. Li and R. McLaren, Environ. Sci. Technol., 2005, 39, 1532-1541.

50 N. Sedehi, H. Takano, V. A. Blasic, K. A. Sullivan and D. O. De Haan, Atmos. Environ., 2013, 77, 656-663.

51 T. B. Nguyen, P. B. Lee, K. M. Updyke, D. L. Bones, J. Laskin, A. Laskin and S. A. Nizkorodov, J. Geophys. Res.: Atmos., 2012, 117, 14.

52 G. T. Drozd and V. F. McNeill, Environ. Sci.: Processes Impacts, 2014, 16, 741-747. 
53 R. Zhao, A. K. Y. Lee, L. Huang, X. Li, F. Yang and J. P. D. Abbatt, Atmos. Chem. Phys., 2015, 15, 6087-6100.

54 P. Lin, J. Laskin, S. A. Nizkorodov and A. Laskin, Environ. Sci. Technol., 2015, 49, 14257-14266.

55 A. Hantzsch, Chem. Ber., 1881, 14, 1637-1638.

56 K. Geibel and F. Strohmer, J. Prakt. Chem., 1991, 333, 895-900.

57 C. Müller-Tautges, A. Eichler, M. Schwikowski and T. Hoffmann, Anal. Bioanal. Chem., 2014, 406, 2525-2532.

58 O. Diels and H. Jost, Ber. Dtsch. Chem. Ges., 1902, 35, 3290-3299.
59 I. Alexandropoulou, T. A. Crabb, A. V. Patel and J. Hudec, Tetrahedron, 1999, 55, 5867-5874.

60 V. Amarnath, D. C. Anthony, K. Amarnath, W. M. Valentine, L. A. Wetterau and D. G. Graham, J. Org. Chem., 1991, 56, 6924-6931.

61 C. Paal, Ber. Dtsch. Chem. Ges., 1884, 17, 2756-2767.

62 L. Knorr, Ber. Dtsch. Chem. Ges., 1884, 17, 2863-2870.

63 A. S. Semeik, M. B. Berez, O. M. Chernova, E. V. Antina, S. A. Syrbu, T. V. Lyubimova and A. M. Kutepov, Russ. Chem. Bull., 2003, 52, 1807-1813.

64 I. Migneault, C. Dartiguenave, M. J. Bertrand and K. C. Waldron, Biotechniques, 2004, 37, 790-802. 